\title{
Presence of Cryptosporidium parvum and Giardia lamblia in water samples from Southeast Asia: towards an integrated water detection system
}

Thulasi Kumar', Mohamad Azlan Abd Majid', Subashini Onichandran', Narong Jaturas ${ }^{1,7}$, Hemah Andiappan ${ }^{1}$, Cristina C. Salibay ${ }^{2}$, Hazel A. L. Tabo², Norbel Tabo², Julieta Z. Dungca ${ }^{3}$, Jitbanjong Tangpong ${ }^{4}$,

Sucheep Phiriyasamith ${ }^{5}$, Boonyaorn Yuttayong ${ }^{6}$, Raxsina Polseela ${ }^{7}$, Binh Nhu Do ${ }^{8}$, Nongyao Sawangjaroen ${ }^{9}$,

Tian-Chye Tan ${ }^{1}$, Yvonne A. L. Lim ${ }^{1}$ and Veeranoot Nissapatorn ${ }^{1 *}$

\begin{abstract}
Background: Access to clean and safe drinking water that is free from pathogenic protozoan parasites, especially Cryptosporidium parvum and Giardia lamblia that cause gastrointestinal illness in humans, is still an issue in Southeast Asia (SEA). This study is the first attempt to detect the aforementioned protozoan parasites in water samples from countries in SEA, using real-time polymerase chain reaction (qPCR) assays.
\end{abstract}

Methods: A total of 221 water samples of 10 I each were collected between April and October 2013 from Malaysia (53), Thailand (120), the Philippines (33), and Vietnam (15). A physicochemical analysis was conducted. The water samples were processed in accordance with the US Environmental Protection Agency's methods 1622/1623.1, microscopically observed and subsequently screened using QPCR assays.

Results: Cryptosporidium oocysts were detected in treated water samples from the Philippines (1/10), with a concentration of $0.06 \pm 0.19$ oocyst/L, and untreated water samples from Thailand (25/93), Malaysia (17/44), and the Philippines (11/23), with concentrations ranging from $0.13 \pm 0.18$ to $0.57 \pm 1.41$ oocyst/L. Giardia cysts were found in treated water samples from the Philippines (1/10), with a concentration of $0.02 \pm 0.06 \mathrm{cyst} / \mathrm{L}$, and in untreated water samples from Thailand (20/93), Vietnam (5/10), Malaysia (22/44), and the Philippines (16/23), with concentrations ranging from $0.12 \pm 0.3$ to $8.90 \pm 19.65$ cyst/L. The pathogens C. parvum and G. lamblia were detected using using qPCR assays by targeting the 138-bp fragment and the small subunit gene, respectively. $C$. parvum was detected in untreated water samples from the Philippines (1/23) and Malaysia (2/44), whilst, G. lamblia detected was detected in treated water samples from the Philippines (1/10) and in untreated water samples from Thailand (21/93), Malaysia (12/44), and the Philippines (17/23). Nitrate concentration was found to have a high positive correlation with (oo)cyst (0.993).

Conclusion: The presence of (oo)cysts in the water samples means that there is potential risk for zoonotic disease transmission in the studied countries. Detection using qPCR is feasible for quantifying both pathogenic C. parvum and G. lamblia in large water samples.

Keywords: Cnyptosporidium parvum, Giardia lamblia, Physicochemical, Microscopy, Real-time polymerase chain reaction, Southeast Asia

\footnotetext{
* Correspondence: veeranoot@um.edu.my

${ }^{1}$ Department of Parasitology (Southeast Asia Water Team), Faculty of

Medicine, University of Malaya, Kuala Lumpur, Malaysia

Full list of author information is available at the end of the article
} 


\section{Background}

Cryptosporidium parvum (C. parvum) and Giardia lamblia (G. lamblia) are protozoan parasites that can cause gastrointestinal illness in humans [1]. Both parasites can be transmitted through water in environments where there are poor sanitation systems, lack of hygiene, an inadequate water management system, and wastewater reuse practices. In recent decades, waterborne outbreaks of cryptosporidiosis and giardiasis have been the most prevalent infections reported in countries such as North America, England, Scotland, and Australia [2]. The existence of protozoans in open water reservoirs and treated water supply is mainly due to the contamination of the environmentally resistant of Cryptosporidium oocyst and Giardia cyst stages. However, the aforementioned condition is highly unaffected in harsh water conditions or disrupted by conventional water disinfection treatments (i.e. chlorination, filtration, etc.) due to its resistance [3].

All over the world, Cryptosporidium spp. and Giardia spp. are two of the highest reported causative parasitic waterborne agents [4], but these two protozoans are especially common waterborne outbreaks in the USA. Cryptosporidiosis and giardiasis can be primarily transmitted via direct contact with contaminated water (diving, swimming, bathing, etc.), [5] via contact with water that has been deficiently treated [6], and via accidental ingestion of water containing (oo)cysts [7]; infection with either can lead to potentially fatal diseases in humans.

The immunomagnetic separation (IMS) technique, which was developed by The United States Environmental Protection Agency (EPA) can be used to morphologically identify both protozoan parasites. However, identification at species-level can only be done using molecular techniques, such as those used in polymerase chain reaction (PCR)-restriction fragment length polymorphism [8, 9] and nested PCR $[10,11]$. These techniques can help to determine the prevalence and contamination level of certain protozoan species, and having this information can then help policymakers put in place preventive measures to eradicate the spread or proliferation of pathogenic species [12]. Generally, untreated water is more likely to be contaminated with protozoan parasites due to poor sanitation, but treated water can also be vulnerable to (oo)cyst contamination, as a result of an inefficient water management system.

This study is the first attempt to detect the aforementioned protozoan parasites in water samples from countries in Southeast Asia (SEA), using real-time PCR (qPCR) assays. Since it was developed in 1992 by Higuchi and coworkers [13], qPCR assay has widely been used for diagnosis in laboratories, and has replaced conventional PCR, which is less sensitive in distinguishing between different species due to slight differences occurring at the nucleotide level. Real-time PCR can detect parasites in large samples (without onsite filtration) over a shorter time period (three hours) $[11,14]$. Previous studies have indicated the successful detection of both parasites using qPCR assays in different assays in different types of water samples (i.e. sewage, swimming pools) [15-17]. The minimum detection limit reported for G. lamblia and $C$. parvum was two cysts and one oocyst, respectively, in 20-1,500 l of spiked water samples [18].

Because the data on water contamination with protozoan parasites is limited, this study aims to examine the current distribution of waterborne protozoan parasites in various types of water samples from four countries in SEA, namely Malaysia, Thailand, the Philippines, and Vietnam. The distribution patterns of $C$. parvum and G. lamblia in the water basins of these countries would indicate if there is potential risk for zoonotic disease transmission in the region.

\section{Methods}

Study site and sampling procedure

A total of 221 water samples were collected between April and October 2013; 53 samples were collected from Malaysia, 120 samples from Thailand, 33 samples from the Philippines, and 15 samples from Vietnam. They comprised both treated and untreated water (see Figs. 1 and 2).

Treated water samples included any drinking water processed via demineralization, reverse osmosis, ozonizations, and UV radiation (i.e. drinking, dispensed, and mineral), as well tap water, which is supplied to buildings after treatment via coagulation, flocculation, sedimentation, filtration, and disinfection. Swimming pool water was also included in the treated water category because pools undergo a treatment process that includes purification, flocculation, filtration, and chemical disinfection (i.e. chlorine, sodium hypochlorite, copper silver ionization, or hydrogen peroxide). In the untreated water category, water samples were collected from rain, springs, wells, recreational water bodies (i.e. lakes, waterfalls, rivers and streams that branch off rivers, such as canals or channels), and effluent water sources.

\section{Physicochemical analysis of the water samples}

Physical parameters (i.e. turbidity, total dissolved solids [TDS], salinity, and dissolved oxygen [DO]) were initially measured in situ using a multiprobe parameter (YSI 556 Multiprobe System, Ohio, USA) at the sampling sites. Meanwhile, chemical parameters (i.e. ammonia, chlorine, nitrate, and nitrite) were measured using a colorimeter (DR/890 Portable Colorimeter, Colorado, USA). Both physical and chemical (physicochemical) parameters were recorded as mean values.

\section{Sample concentration and purification}

Each of water was transported to a laboratory within $24 \mathrm{~h}$ of collection and filtered through a flatbed 


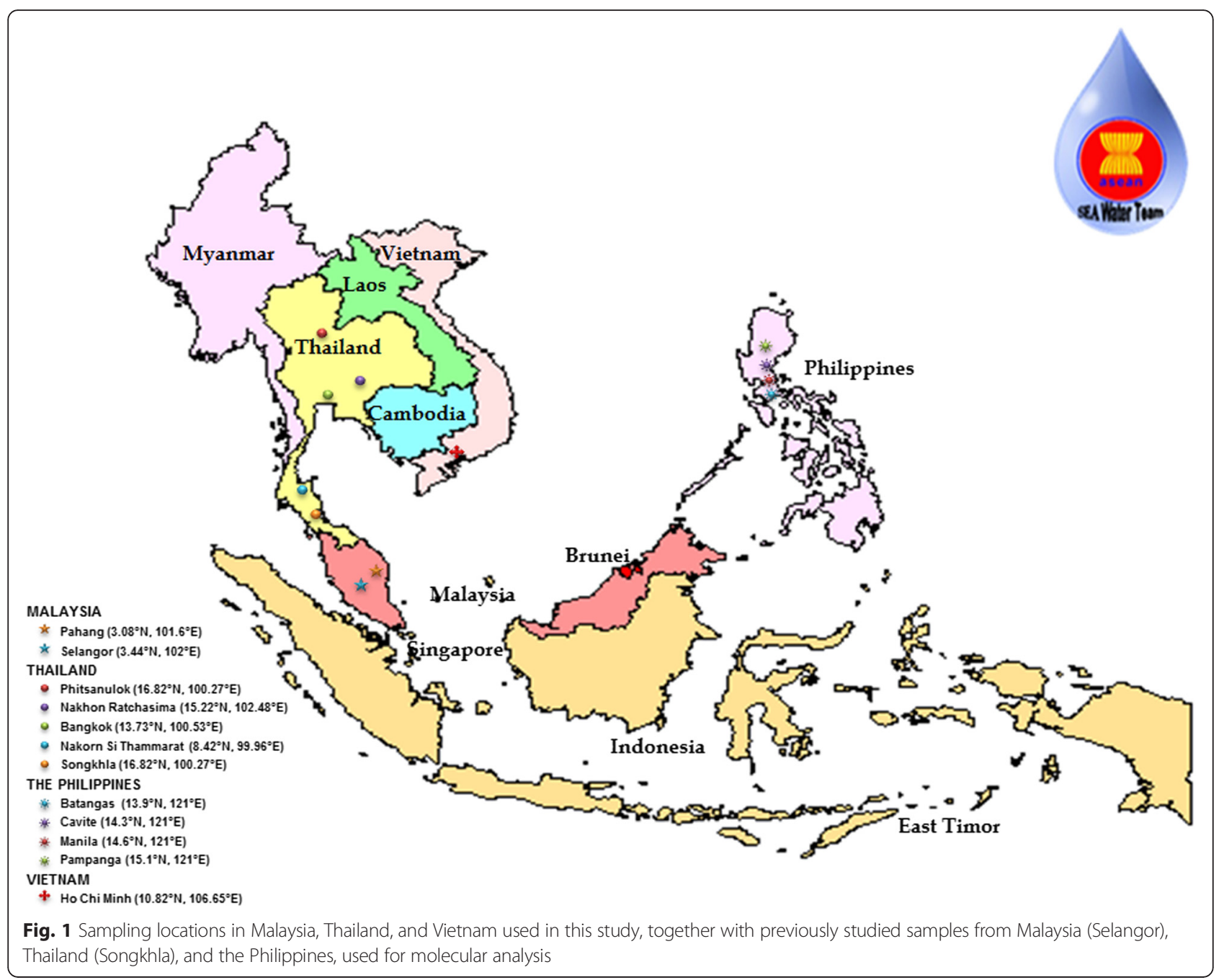

membrane filtration machine (EMD Millipore Corp., Billerica, Massachusettes, USA), using a $142 \mathrm{~mm}$ diameter and a $1.2 \mu \mathrm{m}$ pore-sized nitrocellulose membrane (EMD Millipore Corp., Massachusetts, USA). The accumulated sediments were removed from the top of the membrane using a $0.1 \%$ Tween 80 solution and collected in a Falcon $^{\text {Tx }} 15 \mathrm{ml}$ tube (Brookings, South Dakota, USA). The $10 \mathrm{ml}$ eluate was then subjected to the IMS procedure.

\section{Immunomagnetic separation (IMS) and microscopic examination}

The EPA-established method 1623.1 [19] was employed to detect Cryptosporidium and Giardia (oo)cysts. The eluate was purified using the IMS technique (Dynabeads ${ }^{\circ}$ GC-Combo, Carlsbad, USA). Magnetic beads with Cryptosporidium and Giardia-specific monoclonal antibodies were magnetized, separating the (oo)cyst-magnetic beads complex from the debris [20]. Finally, $50 \mu \mathrm{L}$ of the final product was separated into two portions: one for microscopic examination and the other for molecular analysis.

Each microscopy well slide (Invitrogen Dynal AS, Oslo, Norway) was prepared with $5 \mu \mathrm{l}$ of $1 \mathrm{~N} \mathrm{NaOH}$ solution, and then $25 \mu \mathrm{L}$ of the sample was spotted onto a well. The slide was then stained with fluorescein isothiocyanate-conjugated (FITC) Giardia/Cryptosporidium monoclonal antibody reagent (Cellabs, New South Wales, Australia) and 4', 6'-diamidino-2-phenylindole (DAPI) solution (SigmaAldrich, St. Louis, MO, USA), before being fixed with methanol. The slides were then examined through an epifluorescence microscope (Olympus Model BX-51, Tokyo, Japan), alongside positive (Cellabs, New South Wales, Australia) and negative (purified water) controls. Morphology was determined based on round oocysts (4-6 $\mu \mathrm{m})$ for Cryptosporidium and oval cysts $(9-12 \mu \mathrm{m})$ for Giardia. The numbers of Giardia cysts and Cryptosporidium oocysts were calculated as follows: 


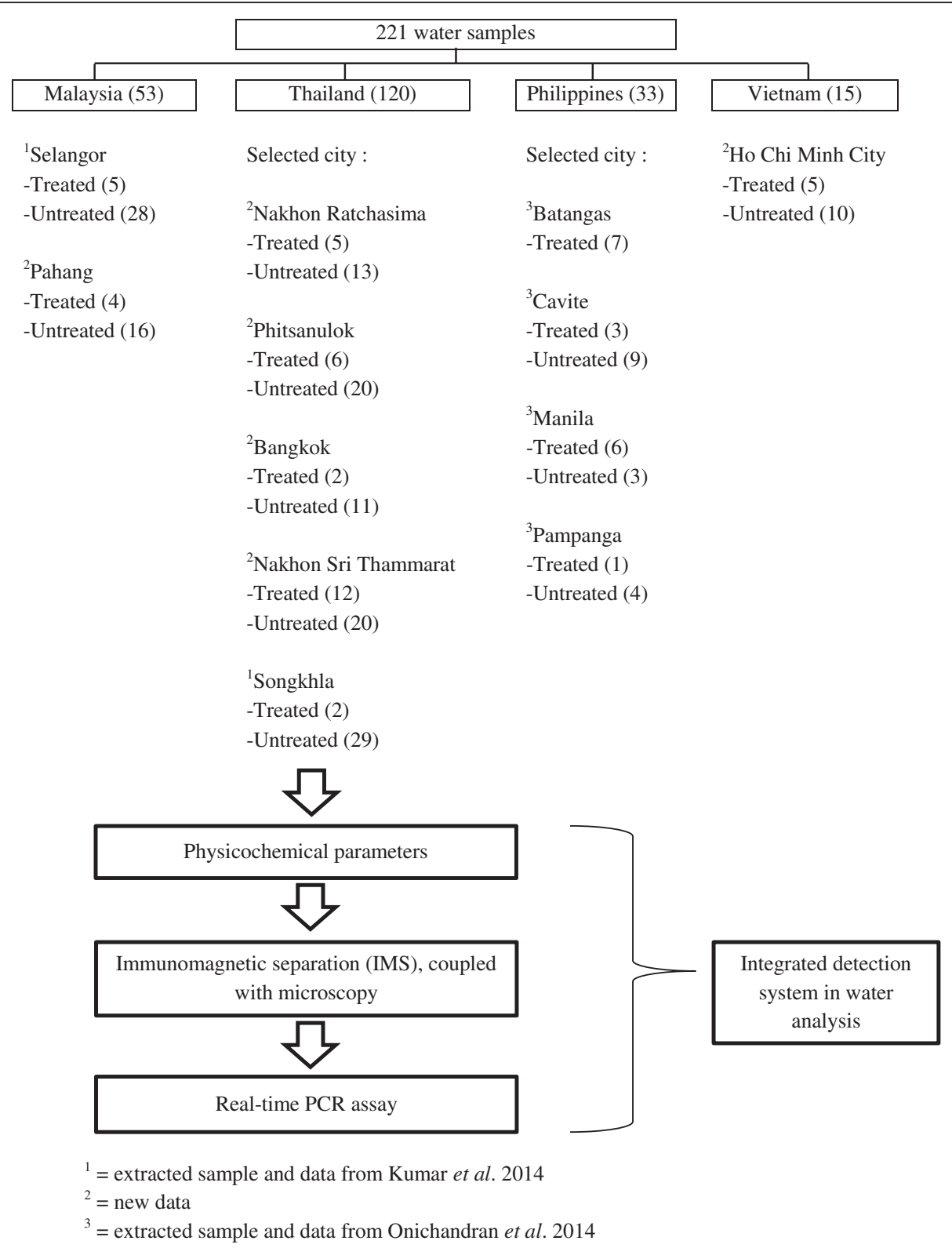

Fig. 2 Flowchart showing the overall analysis of the water samples

number of (oo)cysts per liter $=$ number of (oo)cysts on slide (contained by $25 \mu \mathrm{L}$ after IMS concentration) $/ 10 \mathrm{~L}$ of collected sample.

\section{DNA extraction, purification, and concentration}

The end product of the remaining samples were frozen in liquid nitrogen and thawed in a hot water bath at $56{ }^{\circ} \mathrm{C}$ five times before overnight incubation for $12 \mathrm{~h}$ to break the tough (oo)cyst cell wall. Then, they were subjected to the standard DNA extraction method (QIAamp DNA Mini Kit, Qiagen Inc., Hilden, Germany). All extracted DNA were measured for purity and concentration using a fluorescence spectrophotometer (Micro UV-vis., ES-2 model; Malcom, Tokyo, Japan). The DNA that did not fall within the purity range of 1.7-2.2 were re-concentrated and purified following the protocol for DNA cleanup using Genomic-tips (QIAamp DNA Mini Kit, Qiagen Inc., Hilden, Germany).

\section{Real-time polymerase chain reaction (qPCR)}

The primers and probe sequences for $C$. parvum (AF188110; CrF: 5'-CGC TTC TCT AGC CTT TCA TGA-3', CrR: 5'-CTT CAC GTG TGT TTG CCA AT-3', Cryptosporidium: Texas Red-5' CCA ATC ACA GAA TCA TCA GAA TCG ACT GGT ATC 3'-BHQ2) were 
used to amplify a 138-bp fragment inside the C. parvum specific 452-bp. For G. lamblia, the primers and probe sequences (M54878; Giardia-80 F: 5'-GAC GGC TCA GGA CAA CGG TT-3', Giardia-127R: 5'-TTG CCA GCG GTG TCC G-3', and Giardia 150 T: FAM-5' CCC GCG GCG GTC CCT GCT AG 3'-BHQ1) were chosen to specifically detect a 62-bp fragment within the SSU RNA gene [21, 22].

Singleplex qPCR assays were amplified in a concentration with a volume of $25 \mu \mathrm{L}$, consisting of: PCR premix of $2 \times$ SensiFAST $^{\text {max }}$ Probe No-ROX Kit (Bioline Ltd, London, United Kingdom), 3.75-pmol of each primer, 1.25 -pmol of probe, and $2.5-\mu \mathrm{L}$ of DNA template. Amplification was performed using the CFX96 ${ }^{\mathrm{min}}$ system (Bio-Rad, California, USA), and consisted of five minutes at $95{ }^{\circ} \mathrm{C}$, followed by 45 cycles of $10 \mathrm{~s}$ at $95{ }^{\circ} \mathrm{C}, 20 \mathrm{~s}$ at $55{ }^{\circ} \mathrm{C}$, and $20 \mathrm{~s}$ at $72{ }^{\circ} \mathrm{C}$. Fluorescence was measured during the annealing step of each cycle.

\section{Quality control for qPCR}

The sample's first signal threshold cycle $(\mathrm{Ct})$ value was quantified by comparing it with the standard reference $\mathrm{Ct}$ value of the positive control. The positive control either originated from the G. lamblia axenic culture broth sample (courtesy of Dr. Nongyao Sowangjaroen, Department of Microbiology, Prince of Songkla University, Thailand), or from the seeded Cryptosporidium oocyst samples that were prepared following the EasySeed ${ }^{\text {Tw }}$ Cryptosporidium only procedure (TCS Bioscience Ltd, Buckingham, United Kingdom). At the threshold peak, fluorescence emitted at $490 \mathrm{~nm}$ for $\mathrm{G}$. lamblia and at $575 \mathrm{~nm}$ for C. parvum.

\section{Results}

\section{Baseline data from the physicochemical analysis}

Physicochemical data for both treated and untreated water samples were computed as mean \pm standard deviation (SD), with $95 \%$ confidence intervals (CIs), as shown in Table 1.

The highest ammonia reading was recorded in treated water samples from Malaysia, $2.07 \pm 5.63 \mathrm{mg} / \mathrm{L}$ (CI: 3.90), whereas the highest reading of chlorine was recorded in treated water samples from the Philippines, $0.58 \pm$ $0.74 \mathrm{mg} / \mathrm{L}$ (CI: 0.52 ). Two treated water samples from Thailand showed maximum levels of DO and nitrate, at $5.24 \pm 6.18 \mathrm{mg} / \mathrm{L}$ (CI: 2.64$)$ and $2.31 \pm 8.03 \mathrm{mg} / \mathrm{L}$ (CI: $3.43)$, respectively, whereas untreated water samples from Thailand showed the highest average level of turbidity, at $62.51 \pm 149.91$ NTU (CI: 29.53). Untreated water samples from Vietnam showed maximum levels of TDS (3630.54 $\pm 3546.22 \mathrm{mg} / \mathrm{L} ; \mathrm{CI}: 2197.93)$ and salinity ( $2.77 \pm 3.19 \mathrm{ppt}, \mathrm{CI}: 1.98)$, and treated water samples showed maximum levels of nitrite $(0.58 \pm$ 0.39 mg/L; CI: 0.24).

\section{Detection of Cryptosporidium and Giardia IMS/microscopic identification of Cryptosporidium and Giardia (oo)cysts}

The staining protocol used is based on the concept of conjugated monoclonal antibody with specific FITC dye by targeting the protein of the (oo)cyst cell wall. Meanwhile, DAPI permits the observation of the internal structure of both sporozoites and trophozoites, indicating the viability of individual (oo)cysts [20].

Table 2 shows that Cryptosporidium oocysts were detected in both treated and untreated water samples, from the Philippines (57.8\%; $10 \%$ for treated and $47.8 \%$ for untreated water sample); Malaysia (38.6 \%; $0 \%$ for treated and $38.6 \%$ for untreated water sample); and Thailand (26.9 \%; 0 \% for treated and $26.9 \%$ for untreated water sample). Meanwhile, Giardia cysts were detected in both treated and untreated samples from all four countries: the Philippines (79.6 \%; $10 \%$ for treated and $69.6 \%$ for untreated water sample); Malaysia (50\%; $0 \%$ for treated and $50 \%$ for untreated water sample); Vietnam ( $50 \%$; 0 \% for treated and $50 \%$ for untreated water sample); and Thailand (21.5 \%; 0 \% for treated and $21.5 \%$ for untreated water sample), as shown in Table 2.

\section{Real-time PCR}

The number of cycles required to intersect the threshold level during amplification, which reflects the accumulation of desired fluorescent signals for both C. parvum and G. lamblia, were computed as Ct values. Coefficient of variation $(\mathrm{CV})$ was used to express consistency between cycles as follows:

\section{Formula for $\mathrm{CV}$ of $\mathrm{Ct}$ value $=\underset{\text { Mean of } \mathrm{Ct}}{\text { Standion of } \mathrm{Ct} /}$}

The optimum Ct value for C. parvum was 26.40, compared to a value of 16.26 on the second consecutive amplification cycle and 38.54 on the tenth consecutive amplification cycle. Similarly, for G. lamblia, the optimum Ct value was 28.99, compared to a value of 20.15 on the second amplification cycle and 38.47 on the tenth cycle. The mean values of the 10 consecutive runs were $27.63 \pm$ 7.63 for C. parvum and $29.14 \pm 6.09$ for G. lamblia, as shown in Table 3.

\section{Detection of Cryptosporidium spp. Treated water}

Cryptosporidium oocysts were detected only in treated water samples from the Philippines; $10 \%$ of the samples tested positive with a concentration of $0.06 \pm 0.19$ oocyst/L, as shown in Table 2. None of the positive samples detected using the IMS technique tested positive for $C$. parvum using the qPCR method, as shown in Table 4. 
Table 1 The physicochemical parameter(s) of water samples in selected SEA countries

\begin{tabular}{|c|c|c|c|c|c|c|c|c|c|c|}
\hline \multirow[t]{3}{*}{ Country } & \multirow{3}{*}{$\begin{array}{l}\text { Type of } \\
\text { water }\end{array}$} & \multirow{3}{*}{$\begin{array}{l}\text { Average, SD, and } \\
\mathrm{Cl}(95 \%) \text { values }\end{array}$} & \multicolumn{8}{|c|}{ Water quality parameters (physicochemical) } \\
\hline & & & \multicolumn{4}{|l|}{ Physical } & \multicolumn{4}{|l|}{ Chemical } \\
\hline & & & $\begin{array}{l}\text { Turbidity } \\
\text { (NTU) }\end{array}$ & $\begin{array}{l}\text { TDS } \\
(\mathrm{mg} / \mathrm{L})\end{array}$ & $\begin{array}{l}\text { Salinity } \\
\text { (ppt) }\end{array}$ & $\begin{array}{l}\mathrm{DO} \\
(\mathrm{mg} / \mathrm{L})\end{array}$ & $\begin{array}{l}\text { Chlorine } \\
\text { (mg/L) }\end{array}$ & $\begin{array}{l}\text { Nitrate } \\
(\mathrm{mg} / \mathrm{L})\end{array}$ & $\begin{array}{l}\text { Nitrite } \\
(\mathrm{mg} / \mathrm{L})\end{array}$ & $\begin{array}{l}\text { Ammonia } \\
(\mathrm{mg} / \mathrm{L})\end{array}$ \\
\hline \multirow[t]{6}{*}{ Malaysia } & \multirow[t]{3}{*}{ Treated } & Average & 3.04 & 65.90 & 0.05 & 3.01 & 0.18 & 0.14 & 0.08 & 2.07 \\
\hline & & SD & 5.05 & 88.65 & 0.05 & 3.30 & 0.35 & 0.19 & 0.15 & 5.63 \\
\hline & & $\mathrm{Cl}(95 \%)$ & 3.50 & 61.43 & 0.03 & 2.28 & 0.24 & 0.13 & 0.11 & 3.90 \\
\hline & \multirow[t]{3}{*}{ Untreated } & Average & 17.79 & 58.78 & 0.05 & 2.56 & 0.15 & 0.32 & 0.20 & 0.37 \\
\hline & & SD & 23.34 & 66.48 & 0.04 & 2.15 & 0.16 & 0.41 & 0.38 & 0.51 \\
\hline & & $\mathrm{Cl}(95 \%)$ & 7.96 & 22.68 & 0.01 & 0.73 & 0.05 & 0.14 & 0.13 & 0.17 \\
\hline \multirow[t]{6}{*}{ Thailand } & \multirow[t]{3}{*}{ Treated } & Average & 1.34 & 416.53 & 0.30 & 5.24 & 0.24 & 2.31 & 0.03 & 0.69 \\
\hline & & SD & 1.98 & 1170.86 & 0.92 & 6.18 & 0.76 & 8.03 & 0.04 & 2.90 \\
\hline & & Cl (95\%) & 0.85 & 500.77 & 0.39 & 2.64 & 0.32 & 3.43 & 0.02 & 1.24 \\
\hline & \multirow[t]{3}{*}{ Untreated } & Average & 62.51 & 131.30 & 1.39 & 3.00 & 0.24 & 0.29 & 0.23 & 0.17 \\
\hline & & SD & 149.91 & 198.24 & 4.98 & 1.88 & 0.30 & 0.39 & 1.41 & 0.23 \\
\hline & & Cl (95\%) & 29.53 & 39.05 & 0.98 & 0.37 & 0.06 & 0.08 & 0.28 & 0.05 \\
\hline \multirow[t]{6}{*}{ Philippines } & \multirow[t]{3}{*}{ Treated } & Average & 1.04 & 306.13 & 0.23 & 0.84 & 0.58 & 0.10 & 0.01 & 0.12 \\
\hline & & SD & 1.08 & 254.15 & 0.19 & 0.93 & 0.74 & 0.20 & 0.01 & 0.17 \\
\hline & & $\mathrm{Cl}(95 \%)$ & 0.75 & 176.11 & 0.13 & 0.65 & 0.52 & 0.14 & 0.00 & 0.11 \\
\hline & \multirow[t]{3}{*}{ Untreated } & Average & 8.84 & 530.39 & 0.54 & 0.64 & 0.28 & 0.18 & 0.20 & 0.43 \\
\hline & & SD & 6.38 & 902.59 & 1.00 & 2.28 & 0.50 & 0.25 & 0.32 & 0.32 \\
\hline & & $\mathrm{Cl}(95 \%)$ & 2.61 & 368.87 & 0.41 & 0.93 & 0.20 & 0.10 & 0.13 & 0.13 \\
\hline \multirow[t]{6}{*}{ Vietnam } & \multirow[t]{3}{*}{ Treated } & Average & 0.31 & 224.92 & 0.06 & 4.82 & 0.04 & 0.09 & 0.01 & 0.10 \\
\hline & & SD & 0.38 & 376.10 & 0.06 & 0.98 & 0.06 & 0.05 & 0.01 & 0.01 \\
\hline & & $\mathrm{Cl}(95 \%)$ & 0.37 & 368.58 & 0.06 & 0.96 & 0.06 & 0.05 & 0.01 & 0.01 \\
\hline & \multirow[t]{3}{*}{ Untreated } & Average & 23.60 & 3630.54 & 2.77 & 3.83 & 0.21 & 0.56 & 0.58 & 0.34 \\
\hline & & SD & 21.86 & 3546.22 & 3.19 & 2.28 & 0.17 & 0.42 & 0.39 & 0.29 \\
\hline & & Cl (95\%) & 13.55 & 2197.93 & 1.98 & 1.41 & 0.11 & 0.26 & 0.24 & 0.18 \\
\hline
\end{tabular}

Treated water includes drinking water, water dispenser, mineral water, tap water and swimming pools

Untreated water includes rain water, springs, wells, recreational lake, rivers, waterfalls, canals/channels and effluent water

Table 2 The prevalence of Cryptosporidium and Giardia (oo)cyst under microscopic examination with IMS technique in selected SEA countries

\begin{tabular}{|c|c|c|c|c|c|c|}
\hline \multirow[t]{2}{*}{ Country } & \multirow{2}{*}{$\begin{array}{l}\text { Type of } \\
\text { water }\end{array}$} & \multirow{2}{*}{$\begin{array}{l}\text { No. of } \\
\text { samples }\end{array}$} & \multicolumn{2}{|l|}{ Cryptosporidium } & \multicolumn{2}{|l|}{ Giardia } \\
\hline & & & Positive sample n(\%) & Mean concentration (oocyst/L) & Positive sample n(\%) & Mean concentration (cyst/L) \\
\hline \multirow[t]{2}{*}{ Malaysia } & Treated & 9 & $N / D^{a}$ & N/D & N/D & N/D \\
\hline & Untreated & 44 & $17(38.6)$ & $0.57 \pm 1.41$ & $22(50.0)$ & $0.92 \pm 1.74$ \\
\hline \multirow[t]{2}{*}{ Thailand } & Treated & 27 & N/D & N/D & N/D & N/D \\
\hline & Untreated & 93 & $25(26.9)$ & $0.22 \pm 0.59$ & $20(21.5)$ & $0.12 \pm 0.3$ \\
\hline \multirow[t]{2}{*}{ Philippines } & Treated & 10 & $1(10.0)$ & $0.06 \pm 0.19$ & $1(10.0)$ & $0.02 \pm 0.06$ \\
\hline & Untreated & 23 & $11(47.8)$ & $0.13 \pm 0.18$ & $16(69.6)$ & $8.90 \pm 19.65$ \\
\hline \multirow[t]{2}{*}{ Vietnam } & Treated & 5 & N/D & N/D & N/D & N/D \\
\hline & Untreated & 10 & N/D & N/D & $5(50.0)$ & $0.51 \pm 0.81$ \\
\hline
\end{tabular}

Treated water includes drinking water, water dispenser, mineral water, tap water and swimming pools

Untreated water includes rain water, springs, wells, recreational lake, rivers, waterfalls, canals/channels and effluent water

${ }^{\mathrm{a}} \mathrm{N} / \mathrm{D}$ means not detected 
Table 3 Ct values of C. parvum and G. lamblia after 10 serial dilution in real-time PCR

\begin{tabular}{lll}
\hline Serial dilution & Ct value & \\
\cline { 2 - 3 } & C. parvum & G. lamblia \\
\hline $10^{7}$ & 16.26 & 20.15 \\
$10^{6}$ & 19.18 & 22.21 \\
$10^{5}$ & 21.55 & 24.04 \\
$10^{4}$ & 23.43 & 27.5 \\
Positive control & 26.4 & 28.99 \\
$10^{2}$ & 28.89 & 29.07 \\
$10^{3}$ & 30.55 & 30.25 \\
$10^{8}$ & 35.21 & 34.33 \\
$10^{9}$ & 36.57 & 36.86 \\
$10^{10}$ & 38.54 & 38.47 \\
\hline
\end{tabular}

\section{Untreated water}

Table 2 shows the presence of Cryptosporidium oocysts in untreated water samples from three countries, namely the Philippines (47.8 \%), Malaysia (38.6\%), and Thailand (26.9\%). The highest oocyst concentration was recorded in water samples from Malaysia, with a concentration of $0.57 \pm$ 1.41 oocyst/L, followed by samples from Thailand $(0.22 \pm$ 0.59 oocyst/L) and the Philippines $(0.13 \pm 0.18$ oocyst $/ \mathrm{L})$.

Using the qPCR method, C. parvum was detected in $4.5 \%$ of the samples from Malaysia (Ct: $23.97 \pm 3.8$; CV: $0.16)$ and $4.3 \%$ of the samples from the Philippines (Ct: 36.53), as summarized in Table 4 .

A high presence of oocysts was detected in untreated water samples compared to treated water from the aforementioned countries. C. parvum was found only in untreated water samples from Malaysia and the Philippines.

\section{Detection of Giardia spp.}

\section{Treated water}

Giardia cysts were detected in one treated water sample from the Philippines (10\%), out of 10 total samples, with a concentration of $0.02 \pm 0.06$ cyst/L (see Table 2). Only one sample showed the presence of G. lamblia using the qPCR method (10\%), with a Ct value of $32.26 \pm 0$ (see Table 4).

\section{Untreated water}

Giardia cysts were detected in untreated water samples from the Philippines $(8.90 \pm 19.65$ cyst/L), Malaysia $(0.92 \pm 1.74$ cyst/L), Vietnam $(0.51 \pm 0.81 \mathrm{cyst} / \mathrm{L})$, and Thailand $(0.12 \pm 0.30$ cyst/L), as shown in Table 2 . The Philippines had the highest number of samples positive for G. lamblia (73.9\%), followed by Malaysia (27.3\%) and Thailand $(22.6 \%)$, with $\mathrm{Ct}$ values ranging from 30.49 to 34.41 and CVs ranging from 0.1 to 0.18 (see Table 4). Thus, it can be deducted that Giardia cysts and G. lamblia are frequently detected in untreated water.

\section{Positive correlations between (oo)cyst concentration and physicochemical parameters}

Table 5 shows correlation values between the concentrations of various physicochemical parameters and (oo)cysts.

In Malaysia, TDS detected in treated water samples had a positive correlation with salinity (0.733). Meanwhile, in untreated water samples, salinity had a positive correlation with ammonia (0.657) and TDS (0.708), whereas nitrite also had a high positive correlation with nitrate (0.851). Amongst the parameters, ammonia, nitrate, and nitrite (0.603-0.742) each had a positive correlation with Giardia cyst concentrations in untreated water samples.

In Thailand, the data from treated water samples revealed positive correlations between nitrate and salinity (0.627), nitrate and TDS (0.639), nitrate and nitrite (0.772), and nitrate and chlorine $(0.735)$. Nitrite had a positive correlation with chlorine (0.518), salinity (0.535), TDS (0.55), while salinity showed a positive correlation with TDS (0.998). In untreated water samples, a positive correlation was

Table 4 Ct values observed for real-time PCR assay in selected SEA countries

\begin{tabular}{|c|c|c|c|c|c|c|c|c|}
\hline \multirow[t]{2}{*}{ Country } & \multirow{2}{*}{$\begin{array}{l}\text { Type of } \\
\text { water }\end{array}$} & \multirow{2}{*}{$\begin{array}{l}\text { No. of } \\
\text { samples }\end{array}$} & \multicolumn{3}{|l|}{ C. parvum } & \multicolumn{3}{|l|}{ G. lamblia } \\
\hline & & & Positive sample $\mathrm{n}(\%)$ & Mean Ct value & CV & Positive sample n(\%) & Mean Ct value & CV \\
\hline \multirow[t]{2}{*}{ Malaysia } & Treated & 9 & $\mathrm{~N} / \mathrm{D}^{\mathrm{a}}$ & N/D & N/D & N/D & N/D & N/D \\
\hline & Untreated & 44 & $2(4.5)$ & $23.97 \pm 3.8$ & 0.16 & $12(27.3)$ & $30.49 \pm 5.4$ & 0.18 \\
\hline \multirow[t]{2}{*}{ Thailand } & Treated & 27 & N/D & N/D & N/D & N/D & N/D & N/D \\
\hline & Untreated & 93 & N/D & N/D & $N / D$ & $21(22.6)$ & $32.03 \pm 4.7$ & 0.15 \\
\hline \multirow[t]{2}{*}{ Philippines } & Treated & 10 & N/D & N/D & N/D & $1(10.0)$ & $32.26 \pm 0$ & 0 \\
\hline & Untreated & 23 & $1(4.3)$ & $36.53 \pm 0$ & 0 & $17(73.9)$ & $34.41 \pm 3.5$ & 0.10 \\
\hline \multirow[t]{2}{*}{ Vietnam } & Treated & 5 & N/D & N/D & N/D & N/D & N/D & N/D \\
\hline & Untreated & 10 & $\mathrm{~N} / \mathrm{D}$ & N/D & $\mathrm{N} / \mathrm{D}$ & N/D & N/D & $\mathrm{N} / \mathrm{D}$ \\
\hline
\end{tabular}

Treated water includes drinking water, water dispenser, mineral water, tap water and swimming pools

Untreated water includes rain water, springs, wells, recreational lake, rivers, waterfalls, canals/channels and effluent water

${ }^{\mathrm{a}} \mathrm{N} / \mathrm{D}$ means not detected 
Table 5 Correlation between (oo)cysts concentration and physicochemical parameters

\begin{tabular}{|c|c|c|c|c|c|c|c|c|c|c|c|c|}
\hline \multirow[t]{2}{*}{ Country } & \multirow{2}{*}{$\begin{array}{l}\text { Type of } \\
\text { water }\end{array}$} & \multicolumn{11}{|c|}{ Correlation of (oo)cyst concentration and physicochemical parameter(s) } \\
\hline & & & Oocyst & Cyst & Chlorine & Ammonia & Nitrate & Nitrite & DO & TDS & Salinity & Turbidity \\
\hline \multirow[t]{20}{*}{ Malaysia } & \multirow[t]{10}{*}{ Treated } & Oocyst & 1.000 & & & & & & & & & \\
\hline & & Cyst & 0 & 1.000 & & & & & & & & \\
\hline & & Chlorine & 0 & 0 & 1.000 & & & & & & & \\
\hline & & Ammonia & 0 & 0 & -0.183 & 1.000 & & & & & & \\
\hline & & Nitrate & 0 & 0 & 0.182 & -0.261 & 1.000 & & & & & \\
\hline & & Nitrite & 0 & 0 & -0.171 & -0.155 & -0.284 & 1.000 & & & & \\
\hline & & DO & 0 & 0 & 0.224 & -0.305 & 0.303 & -0.367 & 1.000 & & & \\
\hline & & TDS & 0 & 0 & -0.010 & -0.196 & -0.088 & -0.111 & 0.027 & 1.000 & & \\
\hline & & Salinity & 0 & 0 & -0.344 & 0.181 & -0.433 & -0.113 & -0.488 & 0.733 & 1.000 & \\
\hline & & Turbidity & 0 & 0 & -0.181 & -0.232 & 0.237 & -0.191 & 0.846 & -0.255 & -0.583 & 1.000 \\
\hline & \multirow[t]{10}{*}{ Untreated } & Oocyst & 1.000 & & & & & & & & & \\
\hline & & Cyst & 0.590 & 1.000 & & & & & & & & \\
\hline & & Chlorine & -0.031 & -0.099 & 1.000 & & & & & & & \\
\hline & & Ammonia & 0.421 & 0.742 & 0.181 & 1.000 & & & & & & \\
\hline & & Nitrate & 0.441 & 0.603 & 0.082 & 0.434 & 1.000 & & & & & \\
\hline & & Nitrite & 0.473 & 0.614 & 0.172 & 0.597 & 0.851 & 1.000 & & & & \\
\hline & & DO & -0.022 & -0.078 & -0.307 & -0.317 & -0.126 & -0.326 & 1.000 & & & \\
\hline & & TDS & 0.156 & 0.284 & 0.221 & 0.327 & -0.076 & -0.042 & 0.217 & 1.000 & & \\
\hline & & Salinity & 0.270 & 0.458 & 0.448 & 0.657 & 0.133 & 0.337 & -0.388 & 0.718 & 1.000 & \\
\hline & & Turbidity & -0.043 & -0.081 & 0.228 & -0.005 & 0.040 & -0.027 & -0.292 & 0.226 & 0.240 & 1.000 \\
\hline \multirow[t]{20}{*}{ Thailand } & \multirow[t]{10}{*}{ Treated } & Oocyst & 1.000 & & & & & & & & & \\
\hline & & Cyst & 0 & 1.000 & & & & & & & & \\
\hline & & Chlorine & 0 & 0 & 1.000 & & & & & & & \\
\hline & & Ammonia & 0 & 0 & -0.221 & 1.000 & & & & & & \\
\hline & & Nitrate & 0 & 0 & 0.735 & 0.037 & 1.000 & & & & & \\
\hline & & Nitrite & 0 & 0 & 0.518 & 0.444 & 0.772 & 1.000 & & & & \\
\hline & & DO & 0 & 0 & -0.088 & -0.244 & -0.118 & 0.085 & 1.000 & & & \\
\hline & & TDS & 0 & 0 & -0.027 & 0.236 & 0.639 & 0.550 & -0.087 & 1.000 & & \\
\hline & & Salinity & 0 & 0 & -0.044 & 0.259 & 0.627 & 0.535 & -0.103 & 0.998 & 1.000 & \\
\hline & & Turbidity & 0 & 0 & -0.008 & 0.306 & -0.233 & 0.173 & -0.077 & -0.200 & -0.215 & 1.000 \\
\hline & \multirow[t]{10}{*}{ Untreated } & Oocyst & 1.000 & & & & & & & & & \\
\hline & & Cyst & 0.838 & 1.000 & & & & & & & & \\
\hline & & Chlorine & 0.037 & 0.117 & 1.000 & & & & & & & \\
\hline & & Ammonia & 0.153 & 0.179 & 0.120 & 1.000 & & & & & & \\
\hline & & Nitrate & -0.034 & 0.031 & 0.423 & 0.299 & 1.000 & & & & & \\
\hline & & Nitrite & 0.137 & 0.169 & 0.404 & 0.446 & 0.657 & 1.000 & & & & \\
\hline & & DO & -0.072 & -0.035 & -0.124 & -0.242 & -0.037 & -0.148 & 1.000 & & & \\
\hline & & TDS & -0.147 & -0.176 & 0.262 & -0.252 & 0.090 & 0.162 & 0.200 & 1.000 & & \\
\hline & & Salinity & 0.205 & 0.215 & -0.185 & 0.135 & -0.295 & -0.180 & 0.410 & -0.166 & 1.000 & \\
\hline & & Turbidity & 0.251 & 0.334 & 0.207 & 0.347 & 0.067 & 0.232 & 0.171 & -0.096 & 0.517 & 1.000 \\
\hline \multirow[t]{3}{*}{ Philippines } & \multirow[t]{3}{*}{ Treated } & Oocyst & 1.000 & & & & & & & & & \\
\hline & & Cyst & 1.000 & 1.000 & & & & & & & & \\
\hline & & Chlorine & -0.339 & -0.339 & 1.000 & & & & & & & \\
\hline
\end{tabular}


Table 5 Correlation between (oo)cysts concentration and physicochemical parameters (Continued)

\begin{tabular}{|c|c|c|c|c|c|c|c|c|c|c|c|c|}
\hline & & Ammonia & -0.155 & -0.155 & -0.259 & 1.000 & & & & & & \\
\hline & & Nitrate & 0.993 & 0.993 & -0.260 & -0.178 & 1.000 & & & & & \\
\hline & & Nitrite & 0.272 & 0.272 & -0.146 & -0.414 & 0.199 & 1.000 & & & & \\
\hline & & DO & -0.445 & -0.445 & 0.164 & -0.098 & -0.407 & -0.463 & 1.000 & & & \\
\hline & & TDS & 0.447 & 0.447 & 0.342 & -0.033 & 0.436 & 0.481 & -0.532 & 1.000 & & \\
\hline & & Salinity & 0.447 & 0.447 & 0.338 & -0.048 & 0.435 & 0.497 & -0.539 & 1.000 & 1.000 & \\
\hline & & Turbidity & -0.359 & -0.359 & -0.251 & -0.452 & -0.364 & 0.011 & 0.388 & -0.827 & -0.816 & 1.000 \\
\hline & Untreated & Oocyst & 1.000 & & & & & & & & & \\
\hline & & Cyst & 0.516 & 1.000 & & & & & & & & \\
\hline & & Chlorine & 0.310 & -0.113 & 1.000 & & & & & & & \\
\hline & & Ammonia & 0.297 & 0.450 & 0.330 & 1.000 & & & & & & \\
\hline & & Nitrate & 0.191 & 0.325 & 0.570 & 0.500 & 1.000 & & & & & \\
\hline & & Nitrite & 0.107 & 0.720 & -0.020 & 0.614 & 0.578 & 1.000 & & & & \\
\hline & & DO & -0.354 & 0.084 & -0.879 & -0.507 & -0.533 & -0.016 & 1.000 & & & \\
\hline & & TDS & 0.188 & -0.134 & 0.484 & -0.042 & 0.547 & -0.128 & -0.408 & 1.000 & & \\
\hline & & Salinity & 0.151 & -0.159 & 0.527 & 0.121 & 0.059 & -0.057 & -0.470 & -0.105 & 1.000 & \\
\hline & & Turbidity & 0.337 & 0.127 & 0.600 & 0.690 & 0.288 & 0.146 & -0.596 & 0.138 & 0.252 & 1.000 \\
\hline Vietnam & Treated & Oocyst & 1.000 & & & & & & & & & \\
\hline & & Cyst & 0 & 1.000 & & & & & & & & \\
\hline & & Chlorine & 0 & 0 & 1.000 & & & & & & & \\
\hline & & Ammonia & 0 & 0 & -0.939 & 1.000 & & & & & & \\
\hline & & Nitrate & 0 & 0 & -0.302 & 0.187 & 1.000 & & & & & \\
\hline & & Nitrite & 0 & 0 & 1.000 & -0.937 & -0.312 & 1.000 & & & & \\
\hline & & DO & 0 & 0 & -0.361 & 0.642 & 0.141 & -0.360 & 1.000 & & & \\
\hline & & TDS & 0 & 0 & -0.353 & 0.101 & -0.305 & -0.349 & -0.661 & 1.000 & & \\
\hline & & Salinity & 0 & 0 & -0.287 & -0.022 & -0.086 & -0.286 & -0.779 & 0.960 & 1.000 & \\
\hline & & Turbidity & 0 & 0 & 0.996 & -0.952 & -0.357 & 0.996 & -0.428 & -0.266 & -0.207 & 1.000 \\
\hline & Untreated & Oocyst & 1.000 & & & & & & & & & \\
\hline & & Cyst & 0 & 1.000 & & & & & & & & \\
\hline & & Chlorine & 0 & 0.084 & 1.000 & & & & & & & \\
\hline & & Ammonia & 0 & 0.648 & 0.807 & 1.000 & & & & & & \\
\hline & & Nitrate & 0 & 0.181 & 0.965 & 0.847 & 1.000 & & & & & \\
\hline & & Nitrite & 0 & 0.500 & 0.782 & 0.876 & 0.882 & 1.000 & & & & \\
\hline & & DO & 0 & -0.628 & 0.274 & -0.176 & 0.031 & -0.332 & 1.000 & & & \\
\hline & & TDS & 0 & 0.531 & 0.761 & 0.879 & 0.867 & 0.999 & -0.365 & 1.000 & & \\
\hline & & Salinity & 0 & 0.531 & 0.760 & 0.879 & 0.867 & 0.999 & -0.367 & 1.000 & 1.000 & \\
\hline & & Turbidity & 0 & -0.018 & 0.978 & 0.730 & 0.971 & 0.801 & 0.219 & 0.778 & 0.778 & 1.000 \\
\hline
\end{tabular}

Treated water includes drinking water, water dispenser, mineral water, tap water and swimming pools

Untreated water includes rain water, springs, wells, recreational lake, rivers, waterfalls, canals/channels and effluent water

observed between turbidity and salinity (0.577), and nitrite and nitrate (0.656).

In the Philippines, a positive correlation was observed between nitrate and (oo)cyst concentration (0.993), salinity and (oo)cyst concentration (1), and TDS and (oo)cyst concentration (1) in treated water samples. Positive correlations were also observed between (oo)cyst and cyst concentrations (0.516), salinity and chlorine (0.527), nitrate and TDS (0.547), turbidity and chlorine (0.6), ammonia and nitrite (0.614), ammonia and turbidity (0.69), and nitrite and cyst concentration (0.720) in untreated water samples.

In Vietnam, there was a positive correlation between DO and ammonia (0.642), salinity and TDS (0.96), chlorine 
and turbidity (0.996), and chlorine and nitrite (1) in the treated water samples. In untreated water samples, ammonia was positively correlated with cyst concentration and turbidity, with values of 0.648 and 0.73 , respectively. There was a positive correlation between turbidity and both TDS and salinity (0.778), whereas ammonia had a positive correlation with nitrate, nitrite, and both TDS and salinity, with values of $0.847,0.876$, and 0.879 , respectively. $\mathrm{Ni}-$ trate also showed positive correlation with nitrite $(0.882)$, both TDS and salinity (0.867), and turbidity (0.971), whereas salinity and TDS were both positively correlated with nitrite (0.999).

Overall, positive correlations with the presence of parasites were obtained from the chemicals parameters of ammonia, nitrate, and nitrite from Malaysia, the Philippines, and Vietnam.

\section{Discussion}

In recent years, the SEA region has seen an exponential increase in the number of residents, with an estimated total population of 290.5 million in the four studied countries. The growing population has meant that there is an urgent need for clean water for daily usage, however, the ability to provide a potable water supply is rather limited in some countries due to the costs involved in building dams. Slow economic growth in some countries in SEA has been a limiting factor in preparing, treating, and supplying clean water to the inhabitants. Clean and treated water is readily available in urban areas but in rural areas, people still depend on natural water sources, such as rainwater, rivers, lakes, etc., and these may have become contaminated by waterborne protozoan parasites.

Physicochemical data can be used to preliminary determine water quality, and play a vital role in reflecting nutrient availability which enables parasites to survive. For example, fluctuations in TDS can affect the pH level and DO of the water both directly and indirectly, depending on particle matters. The increase in TDS, and DO are mainly due to increased water usage and precipitation, and are unfavorable conditions for the survival of (oo)cysts, as shown by the negative correlations between (oo)cysts and these parameters in the present study. Previous studies have postulated to use the correlation of nitrate and nitrite concentrations to predict the presence of (oo)cysts in water [20]. Moreover, the present study revealed that ammonia had a positive correlation with oocysts, hence suggesting the need for further evaluate how the aforementioned parameters can be used as indicators of the presence of (oo)cysts in both treated and untreated water samples.

This study showed the existence of an interesting physicochemical relationship between the presence of Cryptosporidium and Giardia. It has been proven that turbidity is positively correlated with a number of (oo)cysts due to run-off intensity and effluent discharge. However, in this study, a negative correlation between (oo)cysts and turbidity was observed. Supporting this, another study has demonstrated that the recovery rate of Cryptosporidium oocysts and Giardia cysts declined as the turbidity level in the water increased, regardless of the filtration method used [23, 24].

Mostly it was the untreated water samples in this study that were contaminated with parasites. The presence of protozoan parasites in waterfalls could explain how humans become directly exposed to parasitic infections (through recreational activities such as bathing and swimming in such waterfalls). Water basins have also been polluted due to high water usage for domestic, fishing, and recreational purposes by local inhabitants living near rivers. Fortunately, there were no Cryptosporidium oocysts and Giardia cysts found in the selected treated water samples from Malaysia, Thailand, and Vietnam. This means that the process of treating water can effectively eliminate (oo)cysts.

In this study, Giardia cysts (cyst/L) appeared to be a major contaminant, and were detected in untreated water samples at a rate of 11.4 times higher than Cryptosporidium oocysts (oocysts/L). This finding is confirmed by the common discovery of Giardia cysts in raw water [25]. This is possibly due to the size of the Giardia cysts (10 to $15 \mu \mathrm{m}$ in length; 7 to $10 \mu \mathrm{m}$ in width) and its thickness (0.3 to $0.5 \mu \mathrm{m}$ ), compared to Cryptosporidium oocysts, as reported by the EPA [26]. Due to their relatively bigger size, these cysts can be easily trapped on filter paper.

In this study, we chose to purify recovered (oo)cysts using filtration and concentrated them via centrifugation without using any chemicals that are potential PCR inhibitors [27, 28]. These inhibitors can affect the amplification by inactivating the thermostable DNA polymerase and/or by interfering with nucleic acids $[29,30]$. Morphological identification was performed through microscopy and molecular screening was conducted using the qPCR method, independently, to obtain a useful and valid comparison between the methods.

Although it has been widely proven that the IMS purification technique is considered to be an efficient step pre-PCR, the presence of foreign particles is inevitable (humus, sediment, etc.) due to poor water quality (cloudy and turbid) [31]. Disturbances of the fluorescent signal generated by bead opacity should be avoided after observation. Both these scenarios will cause interference in the fluorescent signal during PCR assay and lead to inhibiting effects, which are known to decrease PCR efficiency. Thus, more purification steps are necessary for the separation and isolation of (oo)cysts from the slides, which can permit lower (oo)cysts recoveries and DNA yield, prior to molecular analysis. Since there is a low number of Cryptosporidium oocysts and Giardia cysts in environmental samples, even a small loss can have a 
significant influence on their detection via quantitative PCR [32].

Not knowing the time when the (oo)cysts were released into water bodies, the extended time needed for processing samples in the lab until molecular analysis can begin, and the loss that occurs during the physical lysis technique and centrifugation steps might contribute to low recoveries during molecular analysis. These factors could also explain the low level or absence of intact (oo)cysts that are needed to maintain good DNA integral contents [33]. Furthermore, viable (oo)cysts (DNA identification via DAPI staining) were not quantified in this study. Thus, (oo)cysts viably observed by microscopy do not determine the exact quantity confirmed by the qPCR method. In fact, the loss of intact DNA may be due to uncertain conditions such as aged (oo)cysts that were recovered from the samples, distorted (oo)cysts, or (oo)cysts that were removed by the flow of water during sampling, making the concentration of the DNA template below the detection limit for PCR assay.

The IMS technique has been the subject of many studies globally and is considered the gold standard for the identification of (oo)cysts [34]. However, it is also timeconsuming, laborious, and tedious. Moreover, it has a major drawback: cross-reaction with non-target organisms (algae, debris, etc.) and with cysts of other protozoan parasites, in addition to contributing to the loss of (oo)cysts during isolation and purification. The IMS method is also unable to provide specific identification for C. parvum and G. lamblia due to a lack of antibody specificity.

Real-time PCR can provide comparable data to that acquired using the IMS technique without compromising the number of samples. The selection of primer-probe targets is based on the unique molecules of each organism, the biology of which are equally important. Detection of these parasites at a molecular level also takes into account the sensitivity and specificity of each primer-probe used in the PCR method [35, 36]. It is crucial to determine the effectiveness of the analysis in order to avoid crossreactivity among species. The primer-probe specificity for molecular analysis can be tested beforehand for a wide range of protozoan parasites, so that only targeted amplicons are generated prior to sequencing and the DNA sequence of interest can be represented. Although there used to be many types of PCR, such as nested PCR and RFLP sequencing, currently there is a lack of studies published on parasitic DNA detected in water samples of $10 \mathrm{l}$ and above. However, the sensitivity of qPCR is high and able to detect even trace amounts of DNA in an (oo)cyst.

Some environmental factors can also be used to predict the incidence of (oo)cysts in water bodies. For example, meteorological data (i.e. on rainfall) can affect the leachate sediment process after downpour, thus influencing the turbidity and TDS levels of water bodies. Moderate to high correlations were observed between the eight physicochemical parameters employed in this study. Other studies have also pointed to significant interactions of waterborne parasites with other chemical and biological factors $[37,38]$.

\section{Conclusion}

Based on the results obtained, physicochemical parameters can be used to obtain baseline data on the deterioration of water quality and how this is correlated with parasite occurrence (i.e. (oo)cyst). Real-time PCR assay allows for feasible detection and identification of C. parvum oocysts and G. lamblia cysts purified from various types of water samples. The qPCR method can be used for large samples, and is useful for understanding the abundance and distribution of protozoan parasites across different geographic locations. The presence of C. parvum and G. lamblia in both raw and treated water samples can result in potential risk for zoonotic disease transmission in SEA. This requires the urgent attention of relevant policymakers who can implement the necessary preventive measures.

\section{Abbreviations \\ $\mathrm{Cl}$ : confidence interval; Ct: threshold cycle; CV: coefficient of variation; DO: dissolved oxygen; EPA: The US Environmental Protection Agency; IMS: immunomagnetic separation; PCR: polymerase chain reaction; PCR-RFLP: polymerase chain reaction-restriction fragment length polymorph- ism; qPCR: real-time polymerase chain reaction; SD: standard deviation; SEA: Southeast Asia; TDS: total dissolved solids.}

\section{Competing interests}

The authors declare that they have no competing interests.

\section{Authors' contributions}

All authors contributed to the sampling design, selection of locations, and the allocation of laboratory space in each country. VN and YALL were the chief advisors of the project and participated in the study design. TK and MAAM contributed to the water sampling, laboratory work, and writing of the paper. SO, HA, and NJ contributed to the water sampling and laboratory work. NS, CCS, JZD, TCT, VN, and YALL contributed to the writing of the paper. BND, BY, CCS, HALT, JT, JZD, NS, NT, RP, and SP contributed to the water sampling in their respective countries. All authors read and approved the final version of the paper.

\section{Acknowledgments}

The authors would like to thank all the Heads of Departments, staff, and students from Malaysia, Thailand, the Philippines, and Vietnam, who helped us with the water sampling and laboratory work.

\section{Financial support}

This study was supported by the University of Malaya High Impact Research Grant (UM.C/625/1/HIR/MoHE/MED/23 and UM. 0000041/HIR.C3) from the Ministry of Higher Education, Malaysia; postgraduate research grants (PV 049/2011B and PV 014/2012A); and University Malaya Research Grants (UMRG 544/14HTM and UMRG 362/15AFR).

\section{Disclosure}

This work was presented in part at the 13rd International Congress of Parasitology (ICOPA XIII), held in Mexico, 10-15 August 2014 and at 1st International Caparica Conference on Pollutant Toxic and Ionic Molecules (PTIM), held in Portugal, 2-4 November 2015. 


\section{Author details}

'Department of Parasitology (Southeast Asia Water Team), Faculty of Medicine, University of Malaya, Kuala Lumpur, Malaysia. ${ }^{2}$ Biological Science Department, College of Science and Computer Studies, De La Salle University-Dasmariñas, Dasmariñas, Philippines. ${ }^{3}$ School of Science and Technology, Centro Escolar University, Manila, Philippines. ${ }^{4}$ School of Allied Health Sciences and Public Health, Walailak University, Nakhon Si Thammarat, Thailand. ${ }^{5}$ Graduate School, Kasem Bundit University, Bangkok, Thailand. ${ }^{6}$ Regional Medical Sciences Center, Department of Medical Sciences, Ministry of Public Health, Nakhon Ratchasima, Thailand. 'Department of Microbiology and Parasitology, Faculty of Medical Science, Naresuan University, Phitsanulok, Thailand. ${ }^{8}$ Department of Parasitology, Faculty of Medicine, Vietnam Military Medical University, 160 Phung Hung Road, Phuc La Ward, Ha Dong District, Hanoi, Vietnam. ${ }^{9}$ Department of Microbiology, Faculty of Science, Prince of Songkla University, Hat Yai, Thailand.

\section{Received: 25 June 2015 Accepted: 4 January 2016}

\section{Published online: 13 January 2016}

\section{References}

1. Coupe S, Delabre K, Pouillot R, Houdart S, Santillana-Hayat M, Derouin F. Detection of Cryptosporidium, Giardia and Enterocytozoon bieneusi in surface water, including recreational areas: A one-year prospective study. FEMS Immuno Med Microbiol. 2006;47:351-9.

2. Baldursson $S$, Karanis P. Waterborne transmission of protozoan parasites: Review of worldwide outbreaks - An update 2004-2010. Water Res. 2011;45:6603-14.

3. lacovski RB, Barardi CR, Simões CM. Detection and enumeration of Cryptosporidium sp. oocysts in sewage sludge samples from the city of Florianopolis (Brazil) by using immunomagnetic separation combined with indirect immunofluorescence assay. Waste Manag Res. 2004;22:171-6.

4. Karanis $P$, Kourenti C, Smith H. Waterborne transmission of protozoan parasites: A worldwide review of outbreaks and lesson learnt. J Water Health. 2007:5:1-38.

5. Kramer MH, Sorhage FE, Goldstein ST, Dalley E, Wahlquist SP, Herwaldt BL. First reported outbreak in the United States of cryptosporidiosis associated with a recreational lake. Clin Infect Dis. 1998:26:27-33.

6. Betancourt WQ, Rose JB. Drinking water treatment processes for removal of Cryptosporidium and Giardia. Vet Parasitol. 2004;126:219-34.

7. Kramer MH, Herwaldt B, Craun G, Calderon R, Juranek D. Surveillance for waterborne-disease outbreaks-United States, 1993-1994. MMWR CDC Surveill Summ. 1996;45:1-33.

8. Leone A, Ripabelli G, Sammarco ML, Grasso GM. Detection of Cryptosporidium spp from human faeces by PCR-RFLP, cloning and sequencing. Parasitol Res. 2009;104:583-7.

9. Morgan UM, Deplazes P, Forbes DA, Spano F, Hertzberg H, Sargent KD, et al. Sequence and PCR-RFLP analysis of the internal transcribed spacers of the rDNA repeat unit in isolates of Cryptosporidium from different hosts. Parasitology. 1999;118:49-58.

10. Gómez-Couso H, Freire-Santos F, Amar CF, Grant KA, Williamson K, Ares-Mazás ME, et al. Detection of Cryptosporidium and Giardia in molluscan shellfish by multiplexed nested-PCR. Int J Food Microbiol. 2004;91:279-88.

11. Mayer CL, Palmer CJ. Evaluation of $P C R$, nested $P C R$, and fluorescent antibodies for detection of Giardia and Cryptosporidium species in wastewater. Appl Environ Microbiol. 1996:62:2081-5

12. Cacciò SM, Thompson RC, McLauchlin J, Smith HV. Unravelling Cryptosporidium and Giardia epidemiology. Trends Parasitol. 2005;21:430-7.

13. Heid CA, Stevens J, Livak KJ, Williams PM. Real time quantitative PCR. Genome Res. 1996;6:986-94.

14. Alonso JL, Amorós I, Cañigral I. Development and evaluation of a real-time PCR assay for quantification of Giardia and Cryptosporidium in sewage samples. Appl Microbiol Biotechnol. 2011;89:1203-11.

15. Guy RA, Payment P, Krull UJ, Horgen PA. Real-time PCR for quantification of Giardia and Cryptosporidium in environmental water samples and sewage. Appl Environ Microbiol. 2003;69:5178-85.

16. Shields JM, Gleim ER, Beach MJ. Prevalence of Cryptosporidium spp. and Giardia intestinalis in swimming pools, Atlanta, Georgia. Emerg Infect Dis. 2008:14:948-50

17. Moss JA, Gordy J, Snyder RA. Effective concentration and detection of Cryptosporidium, Giardia, and the Microsporidia from environmental matrices. J Pathog. 2014:408204. doi:1011.55/2014/408204.
18. Kaucner C, Stinear T. Sensitive and rapid detection of viable Giardia cysts and Cryptosporidium parvum oocysts in large-volume water samples with wound fiberglass cartridge filters and reverse transcription-PCR. Appl Environ Microbiol. 1998:64:1743-9.

19. USEPA (United States Environmental Protection Agency): Method 1623.1: Cryptosporidium and Giardia in Water by Filtration/IMS/FA. 2012. http:// water.epa.gov/scitech/drinkingwater/labcert/upload/epa816r12001.pdf. Accessed 5 Sept 2015.

20. Onichandran S, Kumar T, Lim YA, Sawangjaroen N, Andiappan H, Salibay CC, et al. Waterborne parasites and physico-chemical assessment of selected lakes in Malaysia. Parasitol Res. 2013:112:4185-91.

21. Verweij JJ, Schinkel J, Laeijendecker D, Rooyen MAAV, Lieshout LV, Polderman AM. Real-time PCR for the detection of Giardia lamblia. Mol Cell Probes. 2003; 17:223-5

22. Verweij JJ, Blangé RA, Templeton $K$, Schinkel J, Brienen EA, van Rooyen MA, et al. Simultaneous Detection of Entamoeba histolytica, Giardia lamblia, and Cryptosporidium parvum in fecal samples by using multiplex real-time PCR. J Clin Microbiol. 2004;42:1220-3.

23. LeChevallier MW, Norton WD. Examining relationships between particle counts and Giardia, Cryptosporidium and turbidity. J Am Water Works Assoc. 1992;84:54-60.

24. Feng $Y$, Zhao $X$, Chen J, Jin W, Zhou X, Li N, et al. Occurrence, source, and human infection potential of Cryptosporidium and Giardia spp. in source and tap water in Shanghai, China. Appl Environ Microbiol. 2011;77:3609-16.

25. Robertson LJ, Gjerde B. Occurrence of Cryptosporidium oocysts and Giardia cysts in raw waters in Norway. Scand J Public Health. 2001:29:200-7.

26. USEPA. (United States Environmental Protection Agency): EPA-822-R-99-008. Drinking Water Health Advisory: Giardia; 1999. http://water.epa.gov/action/ advisories/drinking/upload/2009_02_03_criteria_humanhealth_microbial_ giardiaha.pdf. Accessed 15 Sept 2015.

27. Yu X, Van Dyke MI, Portt A, Huck PM. Development of a direct DNA extraction protocol for real-time PCR detection of Giardia lamblia from surface water. Ecotoxicology. 2009;18:661-8.

28. Bertrand I, Gantzer C, Chesnot T, Schwartzbrod J. Improved specificity for Giardia lamblia cyst quantification in wastewater by development of a real-time PCR method. J Microbiol Methods. 2004;57:41-53.

29. Schriewer A, Wehlmann A, Wuertz S. Improving qPCR efficiency in environmental samples by selective removal of humic acids with DAX-8. J Microbiol Methods. 2011;85:16-21.

30. Abu Al-Soud W, Rådström P. Effects on amplification facilitators on diagnostic PCR in the presence of blood, feces and meat. J Clin Microbiol. 2000;38:4463-70.

31. Fontaine M, Guillot E. Development of a TaqMan quantitative PCR assay specific for Cryptosporidium parvum. FEMS Microbiol Lett. 2002;214:13-7.

32. Adamska M, Leońska-Duniec A, Sawczuk M, Maciejewska A, Skotarczak B. Recovery of Cryptosporidium from spiked water and stool samples measured by PCR and real time PCR. Vet Med. 2012;57:224-32.

33. Ware MW, Keely SP, Villegas EN. Development and evaluation of an off-theslide genotyping technique for identifying Giardia cysts and Cryptosporidium oocysts directly from US EPA Method 1623 slides. J Appl Microbiol. 2013;115:298-309.

34. Fontaine M, Guillot E. An immunomagnetic separation-real-time PCR method for quantification of Cryptosporidium parvum in water samples. J Microbiol Methods. 2003:54:29-36.

35. Tanriverdi S, Tanyeli A, Başlamişli F, Köksal F, Kilinç Y, Feng X, et al. Detection and genotyping of oocysts of Cryptosporidium parvum by real-time PCR and melting curve analysis. J Clin Microbiol. 2002;40:3237-44

36. Amar CF, Dear PH, McLauchlin J. Detection and genotyping by real-time PCR/RFLP analyses of Giardia duodenalis from human faeces. J Med Microbiol. 2003;52:681-3.

37. Bonadonna L, Briancesco R, Ottaviani M, Veschetti E. Occurrence of Cryptosporidium oocysts in sewage effluents and correlation with microbial, chemical and physical water variables. Environ Monit Assess. 2002;75:241-52.

38. Briancesco R, Bonadonna L. An Italian study on Cryptosporidium and Giardia in wastewater, fresh water and treated water. Environ Monit Assess. 2005;104:445-57. 